\title{
Decentralized Coding Algorithms for Distributed Storage in Wireless Sensor Networks
}

\author{
Zhenning Kong, Student Member, IEEE, Salah A. Aly, Member, IEEE, Emina Soljanin, Senior Member, IEEE,
}

\begin{abstract}
We consider large-scale wireless sensor networks with $n$ nodes, out of which $k$ are in possession, (e.g., have sensed or collected in some other way) $k$ information packets. In the scenarios in which network nodes are vulnerable because of, for example, limited energy or a hostile environment, it is desirable to disseminate the acquired information throughout the network so that each of the $n$ nodes stores one (possibly coded) packet so that the original $k$ source packets can be recovered, locally and in a computationally simple way from any $k(1+\epsilon)$ nodes for some small $\epsilon>0$. We develop decentralized Fountain codes based algorithms to solve this problem. Unlike all previously developed schemes, our algorithms are truly distributed, that is, nodes do not know $n, k$ or connectivity in the network, except in their own neighborhoods, and they do not maintain any routing tables.
\end{abstract}

\section{INTRODUCTION}

Wireless sensor networks consist of small devices (sensors) with limited resources (e.g., low CPU power, small bandwidth, limited battery and memory). They are mainly used to monitor and detect objects, fires, temperatures, floods, and other phenomena [1], often in challenging environments where human involvement is limited. Consequently, data acquired by sensors may have short lifetime, and any processing of such data within the network should have low complexity and power consumption [1].

Consider a wireless sensor network with $n$ sensors, where $k$ sensors collect(sense) independent information. Because of the network vulnerability and/or inaccessibility, it is desirable to disseminate the acquired information throughout the network so that each of the $n$ nodes stores one (possibly coded) packet and the original $k$ source packets can be recovered in a computationally simple way from any $k(1+\epsilon)$ of nodes for some small $\epsilon>0$. Two such scenarios are of particular practical interest: to have the information acquired by the $k$ sensors recoverable (1) locally from any neighborhood containing $k(1+\epsilon)$ nodes or (2) from the last $k(1+\epsilon)$ surviving nodes. Fig. 11 illustrates such an example.

Many algorithms have been proposed to solve related distributed storage problems using coding with either centralized or mostly decentralized control. Reed-Solomon based schemes have been proposed in [2]-[5] and Low-Density Parity Check codes based schemes in [6]-[8], and references therein.

Manuscript received January 29, 2009; revised August 7, 2009. Parts of this work were presented in IPSN'08 and ISIT'08 conferences.

Z. Kong is with the Department of Electrical Engineering, Yale University, New Haven, CT 06520, USA, (email: zhenning.kong@yale.edu).

S. A. Aly is with the Department of Computer Science, Texas A\&M University, College Station, TX 77843, USA, (email: salah@cs.tamu.ed).

E. Soljanin is with Bell Laboratories, Alcatel-Lucent, Murray Hill, NJ 07974, USA, (email: emina@lucent.com).

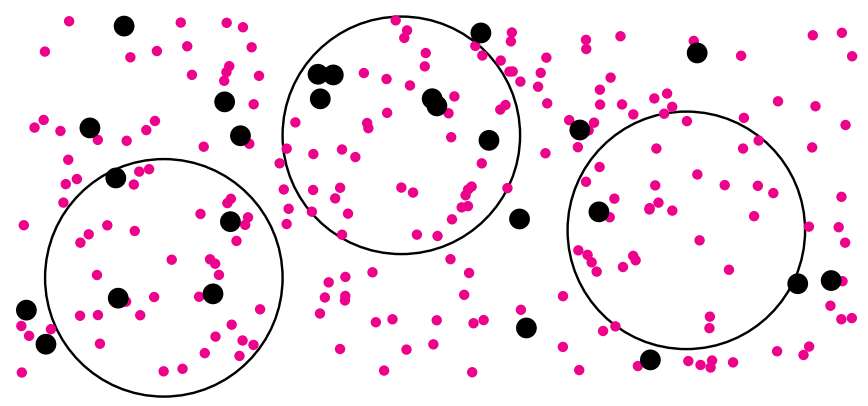

Fig. 1. A sensor network has 25 sensors (big dots) monitoring an area and 225 storage nodes (small dots). A good distributed storage algorithm should enable us to recover the original 25 source packets from any $25+$ nodes (e.g., the set of nodes within any one of the three illustrated circular regions).

Fountain codes have also been considered because they are rateless and because of their coding efficiency and low complexity. In [9] Dimakis el al. proposed a decentralized implementation of Fountain codes using fast random walks to disseminate source data to the storage nodes and geographic routing over a grid, which requires every node to know its location. In [10], Lin et al. proposed a solution employing random walks with stops, and used the Metropolis algorithm to specify transition probabilities of the random walks.

In another line of work, Kamra et al. in [11] proposed a novel technique called growth coding to increase data persistence in wireless sensor networks, that is, the amount of information that can be recovered at any storage node at any time period whenever there is a failure in some other nodes. In [12], Lin et al. described how to differentiate data persistence using random linear codes. Network coding has also been considered for distributed storage in various networks scenarios [13]-[17].

All previous work assumes some access to global information, for example, the total numbers of nodes and sources, which, for large-scale wireless sensor networks, may not be easily obtained or updated by each individual sensor. By contrast, the algorithms proposed in this paper require no global information. For example, in [10], the knowledge of the total number of sensors $n$ and the number of sources $k$ is required to calculate the number of random walks that each source has to initiate, and the probability of trapping data at each sensor. The knowledge of the maximum node degree (i.e., the maximum number of node neighbors) of the graph is also required to perform the Metropolis algorithm. Furthermore, the algorithms proposed in [10] request each sensor to perform encoding only after receiving enough source packets. This demands each sensor to maintain a large temporary memory 
buffer, which may not be practical in real sensor networks.

In this paper, we propose two new algorithms to solve the distributed storage problem for large-scale wireless sensor networks: LT-Codes based distributed storage (LTCDS) algorithm and Raptor Codes based distributed storage (RCDS) algorithm. Both algorithms employ simple random walks. Unlike all previously developed schemes, both LTCDS and RCDS algorithms are truly distributed. That is, except for their own neighborhoods, sensors do not need to know any global information, e.g., the total number of sensors $n$, the number of sources $k$, or routing tables. Moreover, in both algorithms, instead of waiting until all the necessary source packets have been collected to perform encoding, each sensor makes decisions and performs encoding upon each reception of a source packet. This mechanism significantly reduces the node's storage requirements.

The remainder of this paper is organized as follows. In Sec. II we introduce the network and coding model. In Sec. III, we present the LTCDS algorithm and provide its performance analysis. In Sec. IV we present the RCDS algorithm. In Sec. V] we present simulation results for various performance measures of the proposed algorithms

\section{Network AND CODING MODElS}

We model a wireless sensor network consisting of $n$ nodes as a random geometric graph [18], [19], as follows: The nodes are distributed uniformly at random on the plane and all have communication radii of 1 . Thus, two nodes are neighbors and can communicate iff their distance is at most 1 . Among the $n$ nodes, there are $k$ source nodes (uniformly and independently picked from the $n$ ) that have independent information to be disseminated throughout the network for storage. A similar model was considered in [10]. Our algorithms and results apply for many network topologies, e.g., regular grids of [3].

We assume that no node has knowledge about the locations of other nodes and no routing table is maintained; thus the algorithm proposed in [3] cannot be applied. Moreover, we assume that no node has any global information, e.g., the total number of nodes $n$, the total number of sources $k$, or the maximal number of neighbors in the network. Hence, the algorithms proposed in [10] cannot be applied. We assume that each node knows its neighbors. Let $\mathcal{N}(u)$ denote the set of neighbors of $u$. We will refer to the number of neighbors of $u$ as the node degree of $u$, and denote it by $\mu(u)=|\mathcal{N}(u)|$. The mean degree of a graph $G$ is then given by

$$
\bar{\mu}=\frac{1}{|V|} \sum_{u \in G} \mu(u) \text {. }
$$

For $k$ source blocks $\left\{x_{1}, \ldots, x_{k}\right\}$ and a probability distribution $\Omega$ over the set $\{1, \ldots, k\}$, a Fountain code with parameters $(k, \Omega)$ is a potentially limitless stream of output blocks $\left\{y_{1}, y_{2}, \ldots\right\}$ [20], [21]. Each output block is generated by XORing $d$ randomly and independently chosen source blocks, where $d$ is drawn from $\Omega(d)$.

LT (Luby Transform) codes [20], [21] are Fountain codes that employ either the Ideal Soliton distribution

$$
\Omega_{I}(d)= \begin{cases}1 / k, & d=1, \\ 1 /[d(d-1)], & d=2,3, \ldots, k,\end{cases}
$$

or the Robust Soliton distribution, which is defined as follows: Let $R=c_{0} \ln (k / \delta) \sqrt{k}$, where $c_{0}$ is a suitable constant and $0<\delta<1$. Define

$$
\tau(d)= \begin{cases}R / d k, & d=1, \ldots, k / R-1, \\ R \ln (R / \delta) / k, & d=k / R, \\ 0, & d=k / R+1, \ldots, k .\end{cases}
$$

The Robust Soliton distribution is given by

$$
\Omega_{R}(d)=\frac{\tau(d)+\Omega_{I}(d)}{\sum_{i=1}^{k}\left(\tau(i)+\Omega_{I}(i)\right)}, d=1,2, \ldots, k .
$$

Raptor codes are concatenated codes whose inner codes are LT and outer codes are traditional erasure correcting codes. They have linear encoding and decoding complexity [21].

If each node in the network ends up storing an LT or Raptor code output block corresponding to the $k$ source blocks, then the the $k$ source blocks can be recovered in a computationally simple way from any $k(1+\epsilon)$ of nodes for some small $\epsilon>0$, [20], [21]. For different goals, different distributions $\Omega$ may be of interest. Our storage algorithm can take any $\Omega$ as its input.

\section{LT CODES BASED ALgORITHMS}

\section{A. Algorithm Design}

The goal of our storage algorithm is to have each of the $n$ nodes store an LT code output block corresponding to the $k$ input (source) blocks without involvement of a central authority. To achieve this goal, a node in a network would have to store, with probability $\Omega(d)$, a binary sum (XOR) of $d$ randomly and independently chosen source packets. Our main idea to approach this goal in a decentralized way is to (1) disseminate the $k$ source packets throughout the network by $k$ simple random walks and (2) XOR a packet "walking" through a node with a probability $d / k$ where $d$ is chosen at the node randomly according to $\Omega$.

To ensure that each of the $k$ random walks at least once visits each network node, we will let the random walks last longer than the network (graph) cover time [22], [23].

Definition 1: (Cover Time) Given a graph $G$, let $T_{\text {cover }}(u)$ be the expected length of a simple random walk that starts at node $u$ and visits every node in $G$ at least once. The cover time of $G$ is defined by $T_{\text {cover }}(G)=\max _{u \in G} T_{\text {cover }}(u)$.

Lemma 2 (Avin and Ercal [24]): Given a random geometric graph $G$ with $n$ nodes, if it is a connected graph with high probability, then

$$
T_{\text {cover }}(G)=\Theta(n \log n) \text {. }
$$

In addition, the probability that a random walk on $G$ will require more time than $T_{\text {cover }}(G)$ to visit every node of $G$ is $\mathcal{O}(1 / n \log n)$ [22]. Therefore, we can virtually ensure that a random walk visits each network node by requiring that it makes $C_{1} n \log n$ steps for some $C_{1}>0$. To implement this requirement for the $k$ random walks, we set a counter for each source packet and increment it after each transmission. Each time a node receives a packet whose counter is smaller than $C_{1} n \log n$, it accepts the packet for storage with probability $d / k$ (where $d$ is chosen at the node according to $\Omega$ ), and then, regardless of the acceptance decision, it forwards the packet 
to one of its randomly chosen neighbors. Packets older than $C_{1} n \log n$ are discarded.

Note that the above procedure requires the knowledge of $n$ and $k$ at each node. To devise a fully decentralized storage algorithm, we note that each node can observe (1) how often it receives a packets and (2) how often it receives a packets from each source. Naturally, one expects that these numbers depend on the network connectivity $(\mu(u)$ for all $u)$, the size of the graph $n$, and the number of different random walks $k$. We next describe this dependence and show how it can be used to obtain local estimates of global parameters.

The following definitions and claims either come from [22], [23], [25], or can be easily derived based on the results therein.

Definition 3: (Inter-Visit Time) For a random walk on a graph, the inter-visit time of node $u, T_{\text {visit }}(u)$, is the amount of time between any two consecutive visits of the walk to $u$.

Lemma 4: For a node $u$ with node degree $\mu(u)$ in a random geometric graph, the mean inter-visit time is

$$
E\left[T_{\text {visit }}(u)\right]=\bar{\mu} n / \mu(u),
$$

where $\bar{\mu}$ is the mean degree of the graph given by (1).

Lemma 4 implies $n=\mu(u) E\left[T_{\text {visit }}(u)\right] / \bar{\mu}$. While node $u$ can easily measure $E\left[T_{\text {visit }}(u)\right]$, the mean degree $\bar{\mu}$ is a piece of global information and may be hard to obtain. Thus we make a further approximation and let the estimate of $n$ by node $u$ be

$$
\hat{n}(u)=E\left[T_{v i s i t}(u)\right] .
$$

Note that to estimate $n$, it is enough to consider only one of the $k$ random walks. Now to estimate $k$, we also need to consider the $k$ walks jointly without distinguishing between packets originating from different sources.

Definition 5: (Inter-Packet Time) For multiple random walks on a graph, the inter-packet time of node $u, T_{\text {packet }}(u)$, is the amount of time between any two consecutive visits by any of the walks to $u$.

Lemma 6: For a node $u$ with node degree $\mu(u)$ in a random geometric graph with $k$ simple random walks, the mean interpacket time is

$$
E\left[T_{\text {packet }}(u)\right]=\frac{E\left[T_{\text {visit }}(u)\right]}{k}=\frac{\bar{\mu} n}{k \mu(u)},
$$

where $\bar{\mu}$ is the mean degree of the graph given by (1).

Proof: For a given node $u$, each of the $k$ random walks has expected inter-visit time $\frac{\bar{\mu} n}{\mu(u)}$. We now view this process from another perspective: we assume there are $k$ nodes $\left\{v_{1}, \ldots, v_{k}\right\}$ uniformly distributed in the network and an agent from node $u$ following a simple random walk. Then the expected intervisit time for this agent to visit any particular $v_{i}$ is the same as $\frac{\bar{\mu} n}{\mu(u)}$. However, the expected inter-visit time for any two nodes $v_{i}$ and $v_{j}$ is $\frac{1}{k} \frac{\bar{\mu} n}{\mu(u)}$, which gives $(8)$.

Based on Lemmas 4 and 6 , that is equations (6) and (8), we see that each node $u$, can estimate $k$ as

$$
\hat{k}(u)=E\left[T_{\text {visit }}(u)\right] / E\left[T_{\text {packet }}(u)\right] .
$$

We are now ready to state the entire storage algorithm:
Definition 7: (LTCDS Algorithm)

with system parameters $C_{1}, C_{2}>0$ and $\Omega$

\section{Initialization Phase}

Each source node $s, s=1, \ldots, k$

1) attaches a header to its data $x_{s}$, containing its ID and a life-counter $c\left(x_{s}\right)$ set to zero, and then

2) sends its packet to a randomly selected neighbor.

Each node $u$ sets its storage $y_{u}=0$.

Inference Phase (at all nodes $u$ )

1) Suppose $x_{s(u)_{1}}$ is the first source packet that visits $u$, and denote by $t_{s(u)_{1}}^{(j)}$ the time when $x_{s(u)_{1}}$ makes its $j$-th visit to $u$. Concurrently, $u$ maintains a record of visiting times for all packets $x_{s(u)_{i}}$ "walking" through it. Let $t_{s(u)_{i}}^{(j)}$ be the time when source packet $x_{s(u)_{i}}$ makes its $j$-th visit to $u$. After $x_{s(u)_{1}}$ visits $u C_{2}$ times, where $C_{2}>0$ is system parameter, $u$ stops this monitoring and recoding procedure. Denote by $k(u)$ the number of source packets that have visited at least once until that time.

2) Let $J\left(s(u)_{i}\right)$ be the number of visits of source packet $x_{s(u)_{i}}$ to $u$ and let

$$
\begin{aligned}
T_{s(u)_{i}} & =\frac{1}{J\left(s(u)_{i}\right)-1} \sum_{j=1}^{J\left(s(u)_{i}\right)-1}\left(t_{s(u)_{i}}^{(j+1)}-t_{s(u)_{i}}^{(j)}\right) \\
& =\frac{1}{J\left(s(u)_{i}\right)-1}\left(t_{s(u)_{i}}^{\left(J\left(s(u)_{i}\right)\right)}-t_{s(u)_{i}}^{(1)}\right) . \quad(10)
\end{aligned}
$$

Then, the average inter-visit time for node $u$ is

$$
\bar{T}_{v i s i t}(u)=\frac{1}{k(u)} \sum_{i=1}^{k(u)} T_{s(u)_{i}} .
$$

Let $J_{\min }=\min _{s(u)_{i}}\left\{t_{s(u)_{i}}^{(1)}\right\}$ and $J_{\max }=\max _{s(u)_{i}}\left\{t_{s(u)_{i}}^{\left(J\left(s(u)_{i}\right)\right)}\right\}$.

Then the inter-packet time is

$$
\bar{T}_{\text {packet }}(u)=\frac{J_{\max }-J_{\min }}{\sum_{s(u)_{i}} J\left(s(u)_{i}\right)},
$$

and $u$ can estimate $n$ and $k$ as

$$
\hat{n}(u)=\bar{T}_{\text {visit }}(u) \text { and } \hat{k}(u)=\frac{\bar{T}_{\text {visit }}(u)}{\bar{T}_{\text {packet }}(u)} .
$$

3) In this phase, the counter $c\left(x_{s_{i}}\right)$ of each source packet $c\left(x_{s_{i}}\right)$ is incremented by one after each transmission.

Encoding and Storage Phase (at all nodes $u$ )

1) Node $u$ draws $d_{c}(u)$ from $\{1, \ldots, \hat{k}(u)\}$ according to $\Omega$.

2) Upon reciving packet $x$, if $c(x)<C_{1} \hat{n} \log \hat{n}$, node $u$

- puts $x$ into its forward queue and increments $c(x)$.

- with probability $d_{c}(u) / \hat{k}$, accepts $x$ for storage and updates its storage variable $y_{u}^{-}$to $y_{u}^{+}$as

$$
y_{u}^{+}=y_{u}^{-} \oplus x_{s},
$$

If $c(x)<C_{1} \hat{n} \log \hat{n}, x$ is removed from circulation.

3) When a node receives a packet before the current round, it forwards its head-of-line (HOL) packet to a randomly chosen neighbor.

4) Encoding phase ends and storage phase begins when each node has seen its $\hat{k}(u)$ source packets. 


\section{B. Performance Analysis}

Parameters $(k, \Omega)$ determine the error rate performance and encoding/decoding complexity of the corresponding Fountain code. With input $(k, \Omega)$, the LTCDS algorithm produces a distributed Fountain code with parameters $\left(k, \Omega^{\prime}\right)$, where $\Omega^{\prime} \neq \Omega$. We next compute $\Omega^{\prime}$ when the input distribution $\Omega$ is the Robust Soliton (4), and discuss the performance and complexity of the corresponding Fountain code.

Recall that node $u$ draws $d_{c}(u)$ according to $\Omega$, and accepts a passing source packet with probability $d_{c}(u) / k$. Therefore, the number of packets that $u$ accepts, given $d_{c}(u)$, is Binomially distributed with parameter $d_{c}(u) / k$, and the number of packets that $u$ accepts takes value $i$ with probability $\Omega^{\prime}(i)$ :

$$
\begin{aligned}
\Omega^{\prime}(i) & =\sum_{d_{c}(u)=1}^{k} \operatorname{Pr}\left(\tilde{d}_{c}(u)=i \mid d_{c}(u)\right) \Omega\left(d_{c}(u)\right) \\
& =\sum_{d_{c}(u)=1}^{k}\left(\begin{array}{c}
k \\
i
\end{array}\right)\left(\frac{d_{c}(u)}{k}\right)^{i}\left(1-\frac{d_{c}(u)}{k}\right)^{k-i} \Omega\left(d_{c}(u)\right) .
\end{aligned}
$$

A simple way to achieve $\Omega^{\prime}=\Omega$ would be to let each $u$ store each distinct passing source packet until it collects all $k$, and then randomly choose exactly $d_{c}(u)$ packets, where $d_{c}(u)$ is drawn according to $\Omega$, This approach would require large buffers, which is usually not practical, especially when $k$ is large. Therefore, we assume that nodes have limited memory and let them make their decision upon each reception. Our approach, as the following theorem shows, results in a Fountain code with comparable efficiency and the same complexity as the one determined by the Robust or Ideal Soliton distributions.

Theorem 8: Suppose the LTCDS algorithm uses the Robust Soliton distribution (4) for $\Omega$. Then, the $k$ source packets can be recovered from any $K^{\prime}=\beta K$ nodes with probability $1-\delta$ for sufficiently large $k$, where $\beta \geq\left(1-e^{-2}\right)^{-1}$ and $K=$ $k+\mathcal{O}\left(\sqrt{k} \log ^{2}(k / \delta)\right)$ ( $K$ would be sufficient for recovery when $\Omega^{\prime}=\Omega$ ). The decoding complexity is $\mathcal{O}(k \log (k / \delta))$.

Proof: The probability that a node stores no information is

$$
\begin{aligned}
\Omega^{\prime}(0) & =\sum_{d=1}^{k}\left(1-\frac{d}{k}\right)^{k} \Omega(d)<\sum_{d=1}^{k} e^{-d} \Omega(d) \\
& <\sum_{d=1}^{k} \tau(d) e^{-d}+\sum_{d=1}^{k} \Omega_{I}(d) e^{-d} \\
& =\sum_{d=1}^{\frac{k}{R}-1} \frac{R}{k d} e^{-d}+\frac{R \ln \left(\frac{R}{\delta}\right)}{k} e^{-k / R}+\sum_{d=1}^{k} \Omega_{I}(d) e^{-d} \\
& <\frac{R}{k} \sum_{d=1}^{\frac{k}{R}-1} \frac{e^{-1}}{d}+\frac{R \ln \left(\frac{R}{\delta}\right) e^{-\frac{k}{R}}}{k}+\frac{e^{-1}}{k}+\sum_{d=2}^{k} \frac{e^{-2}}{d(d-1)} \\
& <\mathcal{O}\left(\frac{(\ln k)^{2}}{\sqrt{k}}\right)+e^{-2} .
\end{aligned}
$$

Therefore, for sufficiently large $k, \Omega^{\prime}(0)<e^{-2}$. Consequently, if we randomly take $K^{\prime}=\beta K$ nodes from the network, where $\beta \geq\left(1-e^{-2}\right)^{-1}$, we have

$$
\begin{aligned}
\operatorname{Pr}\left\{N_{0}<(1-\alpha)\right. & \left.K^{\prime}\left(1-\Omega^{\prime}(0)\right)\right\} \leq \\
\frac{K^{\prime} \Omega^{\prime}(0)\left(1-\Omega^{\prime}(0)\right)}{\alpha^{2} K^{\prime 2}\left(1-\Omega^{\prime}(0)\right)^{2}} & =\Theta\left(\frac{1}{k}\right),
\end{aligned}
$$

for any $\alpha>0$, where $N_{0}$ denotes the number of nodes that store encoded packets. Therefore, we have $K^{\prime}\left(1-e^{-2}\right) \geq K$ nodes that store encoded packets with a high probability for sufficiently large $n$ and $k$.

We next show that the original $k$ source packets can be recovered based on $K=k+\mathcal{O}\left(\sqrt{k} \log ^{2}(k / \delta)\right)$ stored packets with probability $1-\delta$, by an argument very similar to the one in [20]. When a source packet is decoded (e.g., from stored packets with degree one), we say that all the other encoded packets that contain this source packet are covered. In the decoding process, call the set of covered encoded packets that have not been fully decoded (all the contained source packets are decoded) as the ripple. The main idea of the proof is to show the ripple size variation is very similar to a random walk, and the probability that the ripple size deviates from its mean in $k$ steps by $\Theta(\sqrt{k})$ is small [20].

It can be shown that the expected number of stored packets of degree one is $\theta^{\prime} R$ for some constant $\theta^{\prime}>0$. Employing a Chernoff bound argument, we can show that with probability at least $1-\delta / 3$, the initial ripple size due to degree one packets is at least $\theta R / 2$ for a suitable constant $\theta>0$. Then by the same argument used in the proof for Theorem 17 in [20], it can be shown that without contribution of $\tau(k / R)$ in $\Omega$, the ripple does not disappear for $L=k-1, \ldots, R$ and the decoding process is successful until $R$ stored packets remain undecoded with probability at least $1-\delta / 3$.

Further, like Proposition 15 in [20], we can show that using only the contribution of $\tau(k / R)$ in $\Omega$, the last $R$ blocks can be decoded with probability $1-\delta / 3$ when between $2 R$ and $R$ stored packets remain undecoded. This implies that the decoding process completes successfully with probability $1-\delta$.

Finally, the decoding complexity is the average degree $D$ of a stored packet:

$$
\begin{aligned}
D & =\sum_{i=1}^{k} i\left[\sum_{d=1}^{k}\left(\begin{array}{c}
k \\
i
\end{array}\right)\left(\frac{d}{k}\right)^{i}\left(1-\frac{d}{k}\right)^{k-i} \Omega(d)\right] \\
& =\sum_{d=1}^{k} k\left[\sum_{i=1}^{k}\left(\begin{array}{c}
k-1 \\
i-1
\end{array}\right)\left(\frac{d}{k}\right)^{i}\left(1-\frac{d}{k}\right)^{k-i}\right] \Omega(d) \\
& =\sum_{d=1}^{k} d\left[\sum_{i=0}^{k-1}\left(\begin{array}{c}
k-1 \\
i
\end{array}\right)\left(\frac{d}{k}\right)^{i}\left(1-\frac{d}{k}\right)^{k-1-i}\right] \Omega(d) \\
& =\sum_{d=1}^{k} d \Omega(d)=\mathcal{O}(\log k / \delta)
\end{aligned}
$$

where the last equality is due to Theorem 13 in [20].

From the calculation of $\Omega^{\prime}(0)$, with the Robust or Ideal Soliton distribution, we also have

$$
\Omega^{\prime}(0)>\frac{1}{2 e^{2}} \text {. }
$$


Remark: One interesting implication of (15) and (17) is that in order to achieve the same performance as that of original LT codes, more than $\left(1-e^{-2} / 2\right)^{-1} K \approx 1.07 K$ nodes, but less than $\left(1-e^{-2}\right)^{-1} K \approx 1.15 K$ nodes are required to recover the original $k$ source packets.

Another main performance metric is the transmission cost of the algorithm, which is characterized by the total number of transmissions (the total number of steps of $k$ random walks).

Theorem 9: The total number of transmissions of the LTCDS algorithm is $\Theta(k n \log n)$.

Proof: In the interference phase of the LTCDS algorithm, the total number of transmissions is upper bounded $C^{\prime} n$ for some constant $C^{\prime}>0$. That is because each node needs to receive the first visit source packet for $C_{2}$ times, and by Lemma 4 the mean inter-visit time is $\Theta(n)$. In the encoding phase, in order to guarantee that each source packet visits all the nodes, the number of steps of each of the $k$ random walks is required to be $\Theta(n \log n)$. Since there are $k$ source packets, the total number of transmissions algorithm is $\Theta(k n \log n)$. $\square$

Note that the algorithm proposed in [10] has similar order of total number transmissions. If geometric information is available, as in [9], the complexity can be reduced, e.g., $\Theta(k \sqrt{n} \log n)$ for the algorithm proposed in [9].

\section{Raptor Codes Based Algorithms}

Recall that Raptor codes are concatenated codes whose inner codes are LT and outer codes (pre-codes) are traditional erasure correcting codes. For the pre-codes will use is randomized LDPC codes with $k$ inputs and $m$ outputs $(m \geq k)$. Assume $n$ and $k$ are known or have been estimated at every node. To perform the LDPC coding for $k$ sources in a distributed manner, we again use simple random walks. Each source node first generates $b$ copies of its own source packet, where $b$ follows some distribution $P_{\mathrm{LDPC}}$ defining the LDPC precode. (See [21] for the design of randomized LDPC codes for Raptor codes.) These $b$ copies are then sent into the network by random walks. Each of the remaining $n-k$ nodes in the network chooses to serve as a parity node with probability $(m-k) /(n-k)$. We refer to the parity nodes together with the original (systematic) source nodes as the pre-coding output nodes. All pre-coding output nodes accept a source packet copy with the same probability; consequently, the $b$ copies of a given source packet get distributed uniformly among all pre-coding output nodes. In this way, we have $m$ pre-coding output nodes, each of which contains an XOR of a random number of source packets. The detailed description of the pre-coding algorithm is given below. After obtaining the $m$ pre-coding outputs, to obtain Raptor codes based distributed storage, we apply the LTCDS algorithm with these $m$ nodes as new sources and an appropriate $\Omega$ as discussed in [21].

Definition 10: (Pre-coding Algorithm)

1) Each source node $s, s=1, \ldots, k$ draws a random number $b(s)$ according to the distribution of predefined LDPC codes, generates $b(s)$ copies of its source packet $x_{s}$ with its ID and a counter $c\left(x_{s}\right)$ with an initial value of zero in the packet header, and sends each of them to one of its randomly chosen neighbors.
2) Each of the remaining $n-k$ nodes chooses to serve as a parity node with probability $(m-k) /(n-k)$. These parity nodes and the original source nodes are pre-coding output nodes. Each pre-coding output node $w$ generate a random number $a(w)$ according to the following distribution:

$$
\operatorname{Pr}(a(w)=d)=\left(\begin{array}{l}
k \\
d
\end{array}\right)\left(\frac{E[b]}{m}\right)^{d}\left(1-\frac{E[b]}{m}\right)^{k-d},
$$

where $E[b]=\sum_{b} b P_{\mathrm{LDPC}}(b)$.

3) Each node that has packets in its forward queue before the current round sends its HOL packet to one of its randomly chosen neighbors.

4) When a node $u$ receives a packet $x$ with $c(x)<$ $C_{3} n \log (n), u$ puts the packet into its forward queue and increments the counter.

5) Each pre-coding output node $w$ accepts the first $a(w)$ copies of different $a(w)$ source packet with counters $c(x) \geq C_{3} n \log (n)$, and updates $w$ 's pre-coding result each time as

$$
y_{w}^{+}=y_{w}^{-} \oplus x .
$$

If a copy of $x$ is accepted, it will not be forwarded any more, and $w$ will not accept any other copy of $x_{s_{j}}$. When the node $w$ completes $a(w)$ updates, $y_{w}$ becomes its pre-coding packet.

\section{Performance Evaluation}

We evaluate the performance of LTCDS and RCDS algorithms by simulation. Our main performance metric is the successful decoding probability vs. the query ratio.

Definition 11: The query ratio $\eta$ is the ratio between the number of queried nodes $h$ and the number of sources $k$ :

$$
\eta=h / k \text {. }
$$

Definition 12: (successful decoding) We say that decoding is successful if it results in recovery of all $k$ source packets.

For a query ratio $\eta$, we evaluate $P_{s}$ by simulation as follows: Let $h=\eta k$ denote the number of queried nodes. We select (uniformly at random) $10 \%$ of the $\left(\begin{array}{l}n \\ h\end{array}\right)$ possible subsets of size $h$ of the $n$ network nodes, and try to decode the $k$ source packets from each subset. Then the fraction of times the decoding is successful measures our $P_{s}$.

Fig. 2] shows the decoding performance of LTCDS algorithm with known $n$ and $k$. For $\Omega$, we chose the Ideal Soliton distribution (2). The network is deployed in $\mathcal{A}=[0,5]^{2}$ with density $\lambda=\frac{40}{9}$, and the system parameter $C_{1}=3$. From the simulation results, we can see that when the query ratio is above 2, the successful decoding probability $P_{s}$ is about $99 \%$. When $n$ increases but $k / n$ and $\eta$ remain constant, $P_{s}$ increases when $\eta \geq 1.5$ and decreases when $\eta<1.5$. This is because when there are more nodes, it is more likely that each node has the Ideal Soliton distribution.

In Fig. 3, we fix $\eta$ to 1.4 and 1.7 and $k / n=0.1$. From the results, it can be seen that as $n$ increases, $P_{s}$ increases until it reaches a plateau, which is the successful decoding probability of LT codes. 


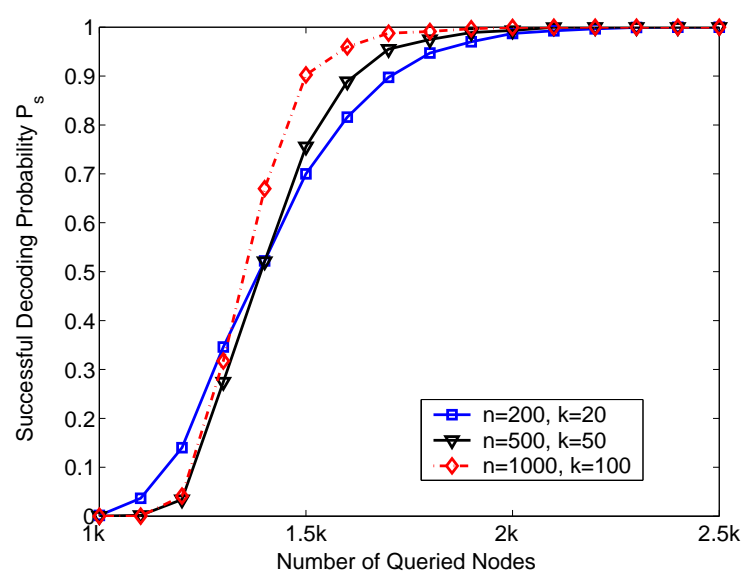

Fig. 2. Performance of LTCDS with known $n$ and $k$ for (a) $n=200, k=20$; (b) $n=500, k=50$; and (c) $n=1000, k=100$.

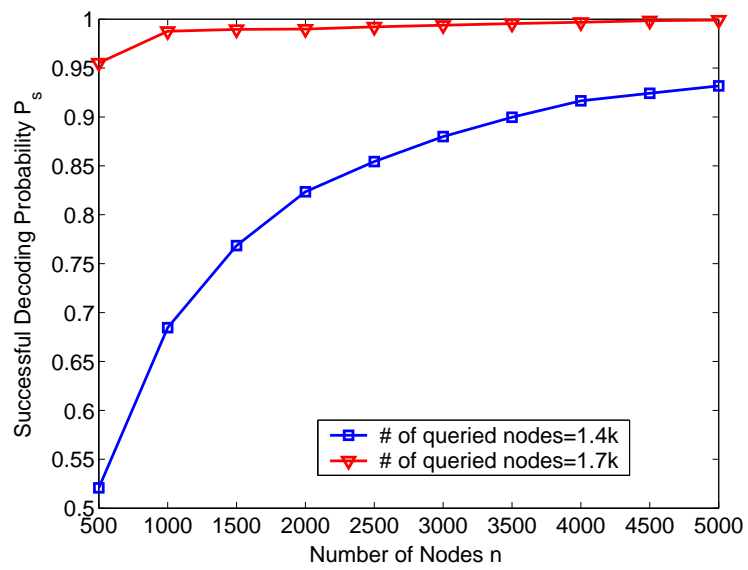

Fig. 3. Performance of LTCDS with different known $n$ and $k$ and fixed number of queried nodes for two cases: (a) $\eta=1.4$; (b) $\eta=1.7$.

We compare the decoding performance of LTCDS with known and unknown values of $n$ and $k$ in Fig. 4 and Fig. 5 . The network is deployed in $\mathcal{A}=[0,5]^{2}$, and the system parameter is set as $C_{1}=10$. To guarantee each node to obtain accurate estimates of $n$ and $k$, we set $C_{2}$ large enough as $C_{2}=50$. The decoding performance of the LTCDS algorithm with unknown $n$ and $k$ is a little bit worse than that of the LTCDS algorithm with known $n$ and $k$ when $\eta$ is small, and almost the same when $\eta$ is large. Such difference between the two algorithms becomes marginal when the number of nodes and sources increase as shown in Fig. 5 .

An interesting observation in Fig. 2. Fig. 4 and Fig. 5 is that the probability of successful decoding is almost zero until we query about $1.1 k$ nodes. This is due to the nodes that store no information in the network. As we pointed out in the Remark after the proof of Theorem 8 , for Robust Soliton distribution, more than $1.07 k$ but less than $1.15 k$ nodes are needed to query to achieve the same performance of LT codes. Similar results also hold for Ideal Soliton distribution.

To investigate how the system parameter $C_{1}$ affects the decoding performance of the LTCDS algorithm with known $n$ and $k$, we fix $\eta$ and vary $C_{1}$. The simulation results are shown in Fig. 6 When $C_{1} \geq 3, P_{s}$ keeps almost like a

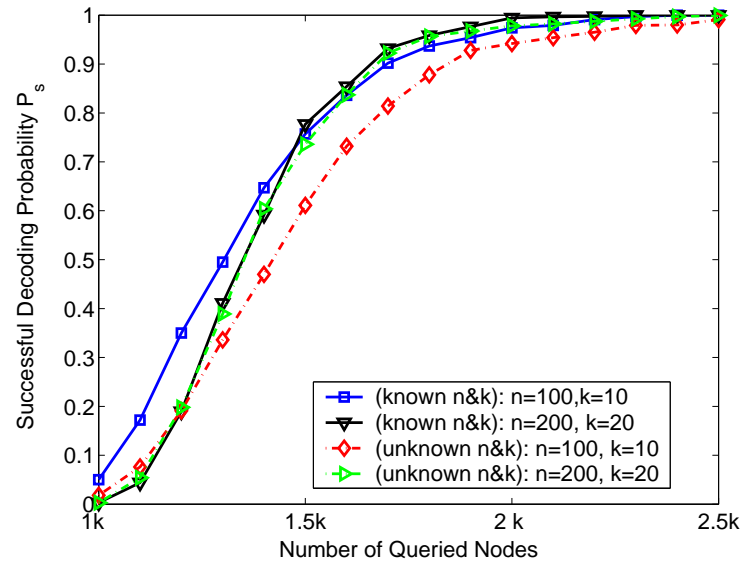

Fig. 4. Performance of LTCDS algorithm with small number of nodes and sources for (a) known $n=100$ and $k=10$; (b) known $n=200$ and $k=20$; (c) unknown $n=100$ and $k=10$; (d) unknown $n=200$ and $k=20$.

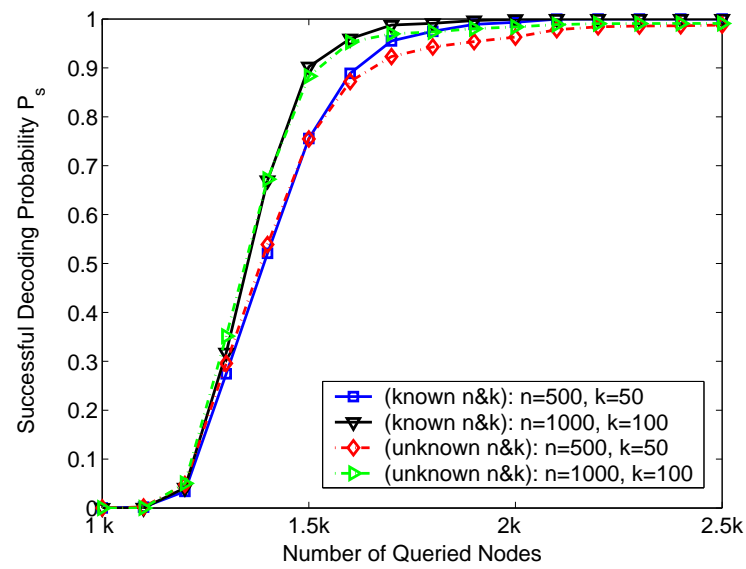

Fig. 5. Performance of LTCDS algorithm with large number of nodes and sources for (a) known $n=500$ and $k=50$; (b) known $n=1000$ and $k=100$; (c) unknown $n=500$ and $k=50$; (d) unknown $n=1000$ and $k=100$.

constant, which indicates that after $3 n \log n$ steps, almost all source packets visit each node at least once.

Furthermore, to investigate how the system parameter $C_{2}$ affects the decoding performance of the LTCDS algorithm, we fix $\eta$ and $C_{1}$, and vary $C_{2}$. From Fig. 7, we can see that when $C_{2}$ is small, the performance of the LTCDS algorithm is very poor. This is due to the inaccurate estimates of $k$ and $n$ by each node. When $C_{2}$ is large, for example, when $C_{2} \geq 30$, the performance is almost the same.

Fig. 8 and Fig. 9 show the histograms of the estimation results for $n$ and $k$, based on equations (13). As expected, the estimates of $k$ are more accurate and concentrated than the estimates of $n$.

\section{REFERENCES}

[1] C. Raghavendra, K. Sivalingam, and T. Znati, Wireless Sensor Networks. Kluwer Academic Publishers, Norwell, MA, USA, 2004.

[2] H. Weatherspoon and J. D. Kubiatowics, "Erasure coding vs. replication: a quantitive comparision," in Proc. of 1st International Workshop on Peer-to-Peer Systems (IPTPS '02), Springer LNCS, Cambridge, MA, USA, , pp. 328-337, March 782002. 


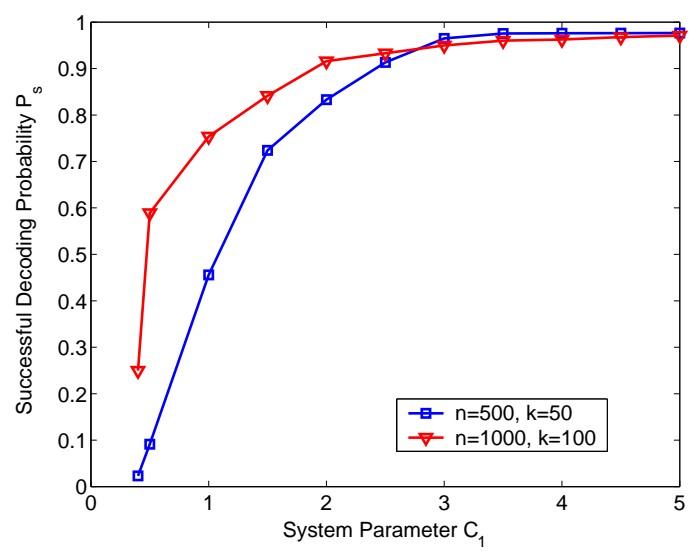

Fig. 6. Performance of LTCDS algorithm with different system parameter $C_{1}$ for two cases: (a) $n=500$ and $k=50$, (b) $n=1000$ and $k=100$.

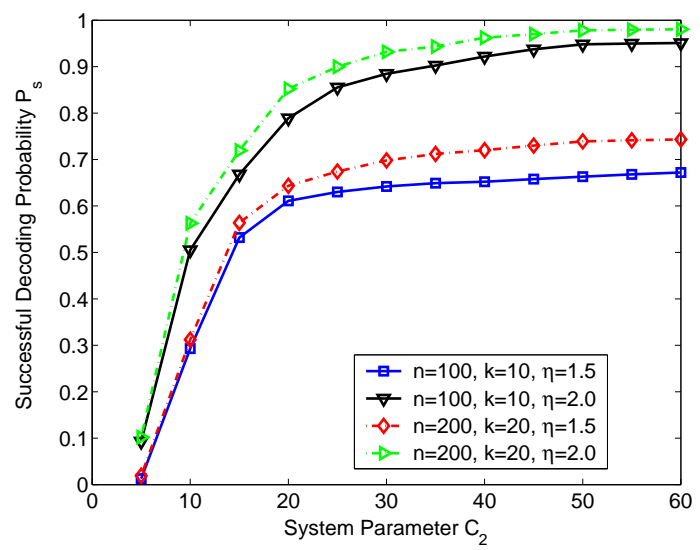

Fig. 7. Performance of LTCDS algorithm with different system parameter $C_{2}$ for (a) $n=100, k=10, \eta=1.5$; (b) $n=100, k=10, \eta=2.0$; (c) $n=200, k=20, \eta=1.5$; (d) $n=200, k=20, \eta=2.0$.

[3] A. G. Dimakis, V. Prabhakaran, and K. Ramchandran, "Ubiquitous access to distributed data in large-scale sensor networks through decentralized erasure codes," in Proc. of 4th IEEE Symposium on Information Processing in Sensor Networks (IPSN '05), Los Angeles, CA, USA, pp. 111-117, April 2005.

[4] A. G. Dimakis, V. Prabhakaran, and K. Ramchandran, "Decentralized erasure codes for distributed networked storage," IEEE Tran. Information Theory, vol. 52, pp. 2809-2816, 2006.

[5] M. Pitkanen, R. Moussa, M. Swany, and T. Niemi, "Erasure codes for increasing the availability of grid data storage," in Proc. of the Advanced International Conference on Telecommunications and International Conference on Internet and Web Applications and Services (AICT/ICIW), pp. 185- 185, 2006.

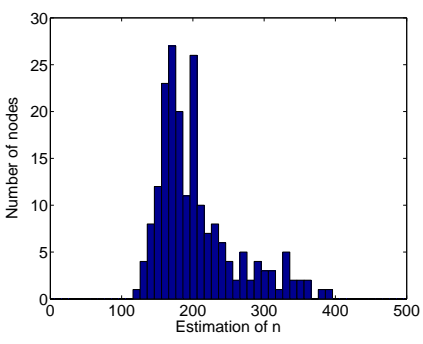

(a)

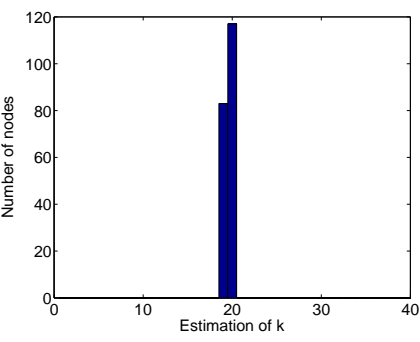

(b)
Fig. 8. Histograms for estimates of $n$ (a) and $k$ (b) in LTCDS algorithm with $n=200$ and $k=20$.

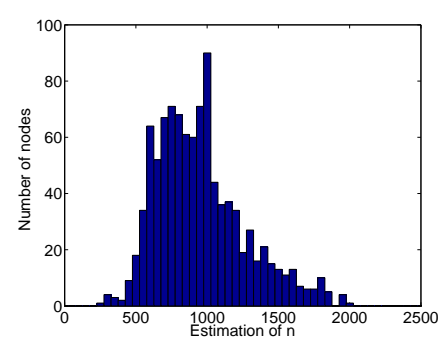

(a)

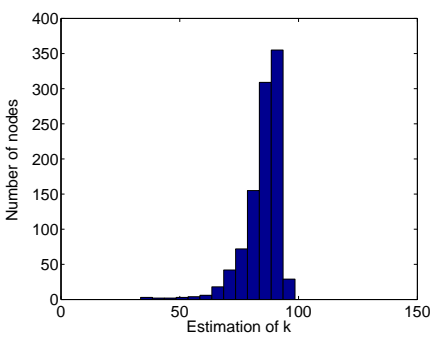

(b)
Fig. 9. Histograms for estimates of $n$ (a) and $k$ (b) in LTCDS algorithm with $n=1000$ and $k=100$.

[6] C. Huang and L. Xu, "Star: An efficient coding scheme for correcting triple storage node failures," in Proc. 4th Usenix conference on file and storage technologies (FAST '05), San Francisco, CA, USA, pp. 15-15, 2005.

[7] J. S. Plank, "Erasure codes for storage applications," in (Tutorial)Proc. 4th Usenix conference on file and storage technologies (FAST '05), San Francisco, CA, USA, 2005.

[8] J. S. Plank and M. G. Thomason, "An exploration of non-asymptotic low-density, parity check erasure codes for wide-area storage applications," Parallel Processing Letters, vol. 17, pp. 103-123, March 2007.

[9] A. G. Dimakis, V. Prabhakaran, and K. Ramchandran, "Distributed fountain codes for networked storage," in Proc. of 31st IEEE International Conference on Acoustics, Speech, and Signal Processing (ICASSP'06), Toulouse, France, May, 2006.

[10] Y. Lin, B. Liang, and B. Li, "Data persistence in large-scale sensor networks with decentralized fountain codes," in Proc. of IEEE INFOCOM'07, Anchorage, AK, USA, pp. 1658-1666, May, 2007.

[11] A. Kamra, V. Misra, J. Feldman, and D. Rubenstein, "Growth codes: Maximizing sensor network data persistence," in Proc. of ACM Sigcom'06, Pisa, Italy, September, 2006.

[12] Y. Lin, B. Li, , and B. Liang, "Differentiated data persistence with priority random linear code," in Proc. of 27th International Conference on Distributed Computing Systems (ICDCS'07), Toronto, Canada, June, 2007.

[13] A. Jiang, "Network coding for joint storage and transmission with minimum cost," in Proc. of IEEE International Symposium on Information Theory (ISIT '06), Seattle, WA, USA, pp. 1359-1363, July, 2006.

[14] D. Wang, Q. Zhang, and J. Liu, "Partial network coding: thoery and application for continuous sensor data collection," in Proc. IEEE 14th international workshop on quality of service (IWQoS), 2006.

[15] S. Acedanski, S. Deb, M. Médard, and R. Koetter, "How good is random linear coding based distributed networked storage?," in Proc. 2nd Workshop on Network Coding (NetCod'05), Pisa, Italy, April, 2005.

[16] A. G. Dimakis, P. B. Godfrey, M. Wainwright, and K. Ramchandran, "Network coding for distributed storage systems," in Proc. of IEEE INFOCOM'07, Anchorage, AK, USA, pp. 2000-2008, May, 2007.

[17] D. Munaretto, J. Widmer, M. Rossi, and M. Zorzi, "Network coding strategies for data persistence in static and mobile sensor networks," in Proc. of International Workshop on Wireless Networks: Communication, Cooperation and Competition (WCN3'07), Limassol, Cyprus, April 2007.

[18] E. N. Gilbert, "Random plane networks," J. Soc. Indust. Appl. Math., vol. 9, pp. 533-543, 1961.

[19] M. Penrose, Random Geometric Graphs. New York: Oxford University Press, 2003.

[20] M. Luby, "LT codes," in 43rd Symposium on Foundations of Computer Science (FOCS 2002), Vancouver, Canada, Nov., 2002.

[21] A. Shokrollahi, "Raptor codes," IEEE Tran. Information Theory, vol. 52, pp. 2551-2567, 2006.

[22] D. Aldous and J. Fill, Reversible Markov Chains and Random Walks on Graphs. Preprint, available at http://statwww.berkeley.edu/users/aldous/RWG/book.html. 2002.

[23] S. Ross, Stochastic Processes. New York: Wiley, second ed., 1995.

[24] C. Avin and G. Ercal, "On the cover time of random geometric graphs," in Proc. 32nd International Colloquium of Automata, Languages and Programming (ICALP'05), Lisboa, Portugal, pp. 677-689, July, 2005.

[25] R. Motwani and P. Raghavan, Randomized Algorithms. Cambridge University Press, 1995. 